\title{
Oxonia-Cope Prins Cyclizations: a Facile Method for the Synthesis of Tetrahydropyranones Bearing Quaternary Centers
}

\author{
Jackline E. Dalgard and Scott D. Rychnovsky* \\ Department of Chemistry, University of California, Irvine, Irvine, CA 92697-2025
}

\section{Supporting Information}

Table of Contents

General Experimental

General Procedure: Synthesis of Silyl Enol Ethers (Method A)

General Procedure: DIBAL-H Reductive Acetylation of Esters

General Procedure: TMSOTf-Mediated Cyclizations

General Procedure: Synthesis of Silyl Enol Ethers (Method B)

\section{Pages}

S2

S6

S7

S8

S11 


\section{General Experiment Details}

All moisture-and air-sensitive reactions were carried out in flame- or oven-dried glassware using magnetic stirring under a positive pressure of argon gas. Standard syringe/septa techniques were employed. Reaction solvents were distilled or obtained from an alumina filtration system when necessary. Thin layer chromatography was performed on Whatman silica gel PE SIL G/UV plates. Concentration of organic solutions was performed using a Büchi rotary evaporator. Flash chromatography was performed on EM Science 230-400 mesh silica gel. Infrared spectra were recorded on a MIDAC Grams/Prospect FT-IR. NMR spectra were recorded on Brüker GN 500, Brüker Omega 500, and Brüker DRX 400 MHz FTNMR instruments. Proton NMR spectra were obtained using $\mathrm{CDCl}_{3}$ as solvent and referenced to residual protiated solvent ( $\left.\delta 7.26 \mathrm{ppm}\right)$ or $\mathrm{C}_{6} \mathrm{D}_{6}$ and referenced to $\delta 7.16 \mathrm{ppm}$. Carbon NMR spectra were recorded in ppm relative to the residual solvent signal: $\mathrm{CDCl}_{3}(\delta 77.0 \mathrm{ppm})$ or $\mathrm{C}_{6} \mathrm{D}_{6}(\delta 128.4 \mathrm{ppm})$. Mass spectra were determined on an AE2-MS 30, a PG 7070E-HF, a CG Analytical 7070E, or a Fisions autospec spectrometer. Optical rotations were measured with a Jasco DIP-370 digital polarimeter. Tetrahydrofuran, ethyl ether and methylene chloride were dried by filtration through alumina according to the procedure by Grubbs. ${ }^{1}$ Capillary GC analysis was performed on a Hewlett Packard Model 6890 instrument equipped with a FID detector. All reagents were purchased from Aldrich Chemical Co. or Acros and were used as received, unless otherwise stated. Elemental analyses were performed by M-H-

\footnotetext{
1 Pangborn, A. B.; Giardello, M. A.; Grubbs, R. H.; Rosen, R. K.; Timmers, F. J. Organometallics
} 1996, 15, 1518-1520. 
W Laboratories (Phoenix, AZ). Structures not numbered in the article were numbered consecutively starting with $\mathbf{1 0 1}$.

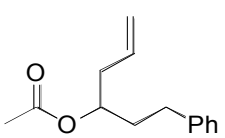

Acetic acid 1-phenethylbut-3-enyl ester (101). Acetic anhydride $(8.2 \mathrm{~mL}, 86.9 \mathrm{mmol}$, 2.0 equiv.), pyridine (5.3 mL, $65.3 \mathrm{mmol}, 1.5$ equiv.) and 4-(dimethylamino)pyridine (1.6 g, 13.1 mmol, 0.3 equiv.) were added to a stirred solution of 1-phenyl-hex-5-en-3-ol (7.7 g, 43.5 mmol, 1.0 equiv.) in $50 \mathrm{~mL}$ of $\mathrm{CH}_{2} \mathrm{Cl}_{2}$. After $5 \mathrm{~h}$, the reaction mixture was diluted with $100 \mathrm{~mL}$ of diethyl ether, washed with $1 \mathrm{~N}$ aq. $\mathrm{HCl}(50 \mathrm{~mL})$, satd. aq. $\mathrm{NaHCO}_{3}(50 \mathrm{~mL})$ and brine $(50 \mathrm{~mL})$. The organic layer was dried over anhydrous $\mathrm{MgSO}_{4}$, and concentrated to provide $8.7 \mathrm{~g}(92 \%)$ of the known acetate ${ }^{2}$ as a colorless oil: IR (neat) 3028, 2951, 1738, 1643, 1373, 1241, $1029 \mathrm{~cm}^{-1} ;{ }^{1} \mathrm{H}$ NMR (400 MHz, $\left.\mathrm{CDCl}_{3}\right) \delta$ 7.38-7.33 (m, 2 H), 7.28-7.23 (m, $3 \mathrm{H}), 5.84$ (dddd, $J=16.9,10.1,7.1,7.1 \mathrm{~Hz}, 1 \mathrm{H})$, 5.20-5.13 (m, 2 H), 5.10-5.03 (m, $1 \mathrm{H}), 2.80-2.64(\mathrm{~m}, 2 \mathrm{H}), 2.43(\mathrm{dd}, J=7.3,7.3 \mathrm{~Hz}, 2$ $\mathrm{H}), 2.11(\mathrm{~s}, 3 \mathrm{H}), 2.00-1.93(\mathrm{~m}, 2 \mathrm{H}) ;{ }^{13} \mathrm{C} \mathrm{NMR}\left(100 \mathrm{MHz}, \mathrm{CDCl}_{3}\right) \delta 170.5,141.3$, $133.4,128.3,128.3,128.2,128.2,125.8,117.6,72.7,38.5,35.1,31.6,21.0$.

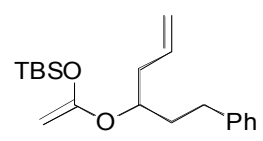

Tert-Butyldimethyl-[1-(1-phenethylbut-3-enyloxy)vinyloxy]silane (8). To a cooled (0 $\left.{ }^{\circ} \mathrm{C}\right)$ solution of diisopropylamine $(0.16 \mathrm{~mL}, 1.12 \mathrm{mmol}, 1.15$ equiv. $)$ was added $n$ butyllithium (0.57 mL, 1.88 $\mathrm{M}$ in hexanes, $1.07 \mathrm{mmol}, 1.1$ equiv.). After $20 \mathrm{~min}$. of stirring, the reaction mixture was cooled to $-78{ }^{\circ} \mathrm{C}$ and the acetate 101 (212 $\mathrm{mg}, 0.97$

\footnotetext{
2 Falck, J. R.; Bhatt, R. K.; Ye, J. J. Am. Chem. Soc. 1995, 117, 5973-5982.
} 
mmol, 1.0 equiv.) was added dropwise via syringe. After 15 min. of stirring at $-78{ }^{\circ} \mathrm{C}$, hexamethylphosphoramide $(0.16 \mathrm{~mL})$ was added followed by tert-butyldimethylsilyl chloride (154 mg, $1.02 \mathrm{mmol}, 1.05$ equiv.). After $30 \mathrm{~min}$. of stirring at $-78{ }^{\circ} \mathrm{C}$, the reaction mixture was slowly warmed to room temperature and the volatiles were removed on a rotary evaporator. Purification was deemed unnecessary and $312 \mathrm{mg}(96 \%)$ of the title compound was isolated as a colorless oil: IR (neat) 3028, 2931, 1651, 1271, 1002, $829 \mathrm{~cm}^{-1} ;{ }^{1} \mathrm{H}$ NMR $\left(400 \mathrm{MHz}, \mathrm{C}_{6} \mathrm{D}_{6}\right)$ d 7.18-7.14 (m, $\left.2 \mathrm{H}\right), 7.11-7.03(\mathrm{~m}, 3 \mathrm{H}), 5.71(\mathrm{~m}$, $1 \mathrm{H}), 5.01-4.97(\mathrm{~m}, 2 \mathrm{H}), 3.96-3.90(\mathrm{~m}, 1 \mathrm{H}), 3.60(\mathrm{~d}, J=2.4 \mathrm{~Hz}, 1 \mathrm{H}), 2.70-2.53(\mathrm{~m}, 2$ H), 2.38-2.21 (m, 2 H), 1.92-1.71 (m, $2 \mathrm{H}), 1.00$ (s, $9 \mathrm{H}), 0.24$ (s, $3 \mathrm{H}), 0.23$ (s, $3 \mathrm{H}) ;{ }^{13} \mathrm{C}$ NMR (125 MHz, $\left.\mathrm{C}_{6} \mathrm{D}_{6}\right) \delta 160.4,142.4,134.6,129.1,128.7,128.2,126.6,117.9,76.4$, $63.2,38.2,35.8,32.3,26.2,18.6,-3.8,-3.9$.

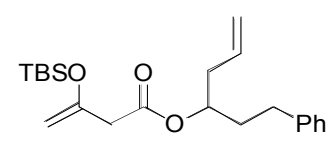

3-(tert-Butyldimethylsilanyloxy)but-3-enoic acid 1-phenethylbut-3-enyl ester (102). A sample of $811 \mathrm{mg}$ of $\mathbf{8}$ was converted to $707 \mathrm{mg}(77 \%)$ of $\mathbf{1 0 2}$, isolated as a colorless oil: IR (neat) 2931, 1740, 1473, 1254, 1030, 840, $699 \mathrm{~cm}^{-1} ;{ }^{1} \mathrm{H}$ NMR (400 MHz, $\left.\mathrm{C}_{6} \mathrm{D}_{6}\right) \delta$ 7.16-7.14 (m, 2 H), 7.09-7.04 (m, 3 H), 5.75-5.63 (m, 1 H), 5.08-4.95 (m, 3 H), 4.23 (s, $1 \mathrm{H}), 4.20$ (s, $1 \mathrm{H}), 2.97$ (s, $2 \mathrm{H}), 2.66-2.45$ (m, $2 \mathrm{H}), 2.27-2.13$ (m, $2 \mathrm{H}), 1.87-1.61$ (m, $2 \mathrm{H}), 0.96$ (s, $9 \mathrm{H}), 0.17(\mathrm{~s}, 3 \mathrm{H}), 0.16(\mathrm{~s}, 3 \mathrm{H}) ;{ }^{13} \mathrm{C} \mathrm{NMR}\left(100 \mathrm{MHz}, \mathrm{C}_{6} \mathrm{D}_{6}\right) \delta$ 169.5, 153.6, 142.3, 134.3, 129.1, 129.1, 129.1, 129.1, 126.6, 118.1, 93.8, 73.4, 43.4, 39.3, 36.1, 32.5, 26.2, 18.6, -4.4, -4.4; HRMS (EI) calcd for $\left[\mathrm{C}_{22} \mathrm{H}_{34} \mathrm{O}_{3} \mathrm{Si}+\mathrm{H}\right]^{+} 375.2355$, found 375.2342 .

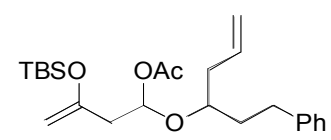




\section{Acetic acid 3-(tert-butyldimethylsilanyloxy)-1-(1-phenethylbut-3-enyloxy)but-3-enyl}

ester (10). A sample of $492 \mathrm{mg}$ of $\mathbf{1 0 2}$ was converted to $536 \mathrm{mg}(98 \%)$ of $\mathbf{1 0}$, isolated as 1:1.3 mixture of diastereomers: IR (neat) 2931, 1741, 1463, 1006, 926, $699 \mathrm{~cm}^{-1} ;{ }^{1} \mathrm{H}$ NMR (500 MHz, $\left.\mathrm{C}_{6} \mathrm{D}_{6}\right) \delta$ 7.20-7.03 (m, $\left.6 \mathrm{H}\right), 6.47-6.43(\mathrm{~m}, 1 \mathrm{H}), 5.92-5.76(\mathrm{~m}, 1 \mathrm{H})$, 5.07-4.98 (m, $2 \mathrm{H}), 4.16(\mathrm{~d}, J=3.7 \mathrm{~Hz}, 1 \mathrm{H}), 4.14(\mathrm{~d}, J=3.3 \mathrm{~Hz}, 1 \mathrm{H}), 3.81-3.73(\mathrm{~m}, 1$ H), 2.86-2.79 (m, 0.4 H), 2.75-2.67 (m, 0.6 H), 2.69-2.51 (m, 3 H), 2.35-2.20 (m, 2 H), 1.87-1.65 (m, 2 H), 1.73 (s, 3 H), 0.98 (s, 3 H), 0.96 (s, 5.4 H), 0.15 (s, 1.2 H), 0.14 (s, $1.2 \mathrm{H}), 0.13$ (s, $1.8 \mathrm{H}), 0.10$ (s, $1.8 \mathrm{H}) ;{ }^{13} \mathrm{C}$ NMR $\left(125 \mathrm{MHz}, \mathrm{C}_{6} \mathrm{D}_{6}\right) \delta$ 170.5, 170.4, 154.9, $142.9,135.5,134.9,129.1,129.1,129.1,129.1,129.0,126.5,126.4,118.0,117.3,96.2$, 95.0, 93.3, 93.0, 79.4, 77.7, 43.8, 43.6, 40.1, 39.4, 37.0, 32.5, 32.1, 26.2, 21.3, 21.2, 18.5, $-4.2,-4.2,-4.6,-4.7$; HRMS (EI) calcd for $\left[\mathrm{C}_{24} \mathrm{H}_{38} \mathrm{O}_{4} \mathrm{Si}+\mathrm{Na}\right]^{+}$441.2437, found 441.2447.

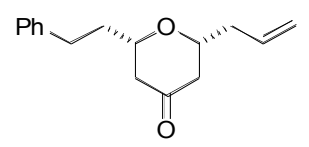

2-Allyl-6-phenethyltetrahydropyran-4-one (17). A sample of $40 \mathrm{mg}$ of $\mathbf{1 0}$ was converted to $23 \mathrm{mg}$ (99\%) of 17, isolated as a colorless oil: IR (neat) 2928, 1720, 1455, $1060 \mathrm{~cm}^{-1} ;{ }^{1} \mathrm{H}$ NMR (400 MHz, $\left.\mathrm{C}_{6} \mathrm{D}_{6}\right) \delta$ 7.19-7.15 (m, $\left.2 \mathrm{H}\right), 7.11-7.02(\mathrm{~m}, 3 \mathrm{H}), 5.75$ (dddd, $J=17.0,10.2,7.0,7.0 \mathrm{~Hz}, 1 \mathrm{H}), 5.06-4.94(\mathrm{~m}, 2 \mathrm{H}), 3.16-3.05(\mathrm{~m}, 2 \mathrm{H}), 2.70-$ 2.48 (m, 2 H), 2.18-1.93 (m, 4 H), 1.86-1.74 (m, 2 H), 1.74-1.62 (m, 1 H), 1.46-1.34

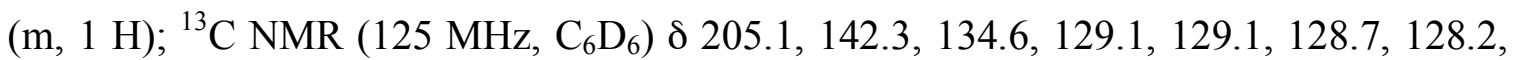
126.6, 117.9, 76.5, 76.1, 48.0, 47.6, 41.1, 38.6, 32.1. HRMS (EI) calcd for $\left[\mathrm{C}_{16} \mathrm{H}_{20} \mathrm{O}_{2}+\mathrm{Na}\right]^{+}$267.1361, found 267.1362. Anal. Calcd for $\mathrm{C}_{16} \mathrm{H}_{20} \mathrm{O}_{2}$ : C, 78.65; H, 8.25. Found: C, 78.44; H, 8.14. 
General Procedure for the Synthesis of Silyl Enol Ethers (Method A):

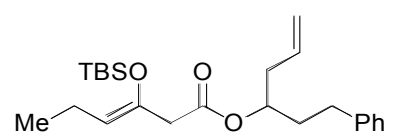

3-(tert-Butyldimethylsilanyloxy)-hex-3-enoic acid 1-phenethylbut-3-enyl ester (103).

Butyryl chloride $(0.2 \mathrm{~mL}, 2.0 \mathrm{mmol}, 1.7$ equiv. $)$ was cooled to $0{ }^{\circ} \mathrm{C}$ in $\mathrm{THF}(2.0 \mathrm{~mL})$. Silyl ketene acetal 8 (392 mg, $1.18 \mathrm{mmol}, 1.0$ equiv.) was added, followed by triethylamine $(0.28 \mathrm{~mL}, 2.0 \mathrm{mmol}, 1.7$ equiv.). The reaction mixture was slowly warmed to room temperature and allowed to stir at this temperature for $16 \mathrm{~h}$. The reaction mixture was then diluted with diethyl ether $(20 \mathrm{~mL})$ and washed with water $(2 \times 5 \mathrm{~mL})$. The organic layer was dried over anhydrous $\mathrm{MgSO}_{4}$ and concentrated to provide a yellow oil. Purification $\left(\mathrm{SiO}_{2}, 1: 9\right.$ ether/hexanes, $\left.1.5 \% \mathrm{Et}_{3} \mathrm{~N}, \mathrm{R}_{\mathrm{f}}=0.38\right)$ provided $407 \mathrm{mg}(86 \%)$ of 103 (single $Z$-alkene isomer by NOE studies) as a colorless oil: IR (neat) 2959, 1738, 1362, 1254, 1152, $839 \mathrm{~cm}^{-1} ;{ }^{1} \mathrm{H}$ NMR (500 MHz, $\left.\mathrm{C}_{6} \mathrm{D}_{6}\right) \delta 7.18-7.13(\mathrm{~m}, 2 \mathrm{H}), 7.10-7.03$ (m, $3 \mathrm{H}), 5.74-5.65(\mathrm{~m}, 1 \mathrm{H}), 5.06-4.95(\mathrm{~m}, 3 \mathrm{H}), 4.60(\mathrm{t}, J=7.0 \mathrm{~Hz}, 1 \mathrm{H}), 2.96(\mathrm{~s}, 2 \mathrm{H})$, 2.66-2.59 (m, $1 \mathrm{H}), 2.55-2.46(\mathrm{~m}, 1 \mathrm{H}), 2.25-2.08(\mathrm{~m}, 4 \mathrm{H}), 1.81-1.76(\mathrm{~m}, 1 \mathrm{H}), 1.72-$ $1.64(\mathrm{~m}, 1 \mathrm{H}), 0.98(\mathrm{~s}, 9 \mathrm{H}), 0.94(\mathrm{t}, J=7.5 \mathrm{~Hz}, 3 \mathrm{H}), 0.15(\mathrm{~s}, 3 \mathrm{H}), 0.14(\mathrm{~s}, 3 \mathrm{H}) ;{ }^{13} \mathrm{C}$ NMR (125 MHz, $\left.\mathrm{C}_{6} \mathrm{D}_{6}\right) \delta 169.9,144.0,142.3,134.4,129.1,129.1,128.7,126.6,118.1$, 114.6, 73.4, 43.6, 39.3, 36.2, 32.4, 26.3, 19.6, 18.8, 14.8, -3.6, -3.6; HRMS (EI) calcd for $\left[\mathrm{C}_{24} \mathrm{H}_{38} \mathrm{O}_{3} \mathrm{Si}+\mathrm{H}\right]^{+}$403.2668, found 403.2650.

\section{General Procedure for the DIBAL-H Reductive Acetylation of Esters:}




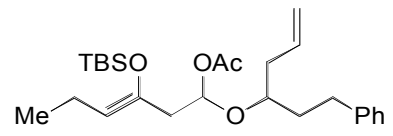

\section{Acetic acid 3-(tert-butyldimethylsilanyloxy)-1-(1-phenethylbut-3-enyloxy)hex-3-enyl}

ester (11). Diisobutylaluminum hydride $(0.45 \mathrm{~mL}, 447 \mu \mathrm{L}, 1.5$ equiv.) was added to a cooled $\left(-78{ }^{\circ} \mathrm{C}\right)$ solution of the ester $103\left(120 \mathrm{mg}, 298 \mu \mathrm{mol}, 1.0\right.$ equiv.) in $\mathrm{CH}_{2} \mathrm{Cl}_{2}(1.8$ $\mathrm{mL}$ ). After $1.5 \mathrm{~h}$, the ester was consumed (monitored by LRMS) and pyridine (60 $\mu \mathrm{L}$, $745 \mu$ mol, 2.5 equiv.) was added, followed by 4-(dimethylamino)pyridine (91 $\mathrm{mg}$ in 1 $\mathrm{mL}$ of $\mathrm{CH}_{2} \mathrm{Cl}_{2}, 745 \mu \mathrm{mol}, 2.5$ equiv.) and acetic anhydride (141 $\mu \mathrm{L}, 1.49 \mathrm{mmol}, 5.0$ equiv.). The reaction mixture was stirred for $16 \mathrm{~h}$ at $-78{ }^{\circ} \mathrm{C}$ then slowly warmed to $0{ }^{\circ} \mathrm{C}$ over $3 \mathrm{~h}$. The reached mixture was carefully quenched with Rochelle's salt (2 mL) and water $(2 \mathrm{~mL})$ then extracted with diethyl ether $(3 \times 10 \mathrm{~mL})$. The organic extracts were washed with satd. aq. $\mathrm{NaHCO}_{3}(5 \mathrm{~mL})$ and brine $(50 \mathrm{~mL})$ then dried over anhydrous $\mathrm{MgSO}_{4}$, and concentrated to provide $130 \mathrm{mg}$ (98\%) of $\alpha$-acetoxy ether $\mathbf{1 1}$ as a colorless oil as a 1:1 mixture of diastereomers: IR (neat) 2932, 1741, 1373, 1007, $838 \mathrm{~cm}^{-1} ;{ }^{1} \mathrm{H}$ NMR (400 MHz, $\left.\mathrm{C}_{6} \mathrm{D}_{6}\right) \delta$ 7.23-7.20 (m, $\left.4 \mathrm{H}\right), 7.16-7.13(\mathrm{~m}, 6 \mathrm{H}), 6.48(\mathrm{t}, J=5.4 \mathrm{~Hz}, 1$ $\mathrm{H}), 6.46(\mathrm{t}, J=5.0 \mathrm{~Hz}, 1 \mathrm{H}), 5.97-5.82(\mathrm{~m}, 2 \mathrm{H}), 5.10-5.00(\mathrm{~m}, 4 \mathrm{H}), 4.62(\mathrm{t}, J=7.1 \mathrm{~Hz}$, $2 \mathrm{H}), 3.84-3.75(\mathrm{~m}, 2 \mathrm{H}), 2.91-2.74(\mathrm{~m}, 2 \mathrm{H}), 2.71-2.51(\mathrm{~m}, 6 \mathrm{H}), 2.42-2.22(\mathrm{~m}, 4 \mathrm{H})$, 2.16-2.08 (m, $4 \mathrm{H}), 1.93-1.78(\mathrm{~m}, 4 \mathrm{H}), 1.73(\mathrm{~s}, 3 \mathrm{H}), 1.72(\mathrm{~s}, 3 \mathrm{H}), 1.03(\mathrm{~s}, 9 \mathrm{H}), 1.02(\mathrm{~s}$, $9 \mathrm{H}), 0.97$ (t, $J=7.4 \mathrm{~Hz}, 3 \mathrm{H}), 0.93(\mathrm{t}, J=7.5 \mathrm{~Hz}, 3 \mathrm{H}), 0.18(\mathrm{~s}, 3 \mathrm{H}), 0.18(\mathrm{~s}, 3 \mathrm{H}), 0.14$ (s, $3 \mathrm{H}), 0.13(\mathrm{~s}, 3 \mathrm{H}) ;{ }^{13} \mathrm{C} \mathrm{NMR}\left(125 \mathrm{MHz}, \mathrm{C}_{6} \mathrm{D}_{6}\right) \delta 170.5,170.4,145.2,145.2,143.0$, $142.9,135.5,134.9,129.1,129.1,129.0,129.0,129.0,129.0,126.5,126.4,126.4,117.9$, $117.3,114.6,114.3,96.7,95.3,79.4,77.8,43.7,43.5,40.2,39.2,36.9,32.5,31.9,31.9$, 
26.3, 26.3, 21.3, 21.2, 19.6, 19.6, 18.8, 18.8, 14.9, 14.9, -3.4, -3.6; HRMS (EI) calcd for $\left[\mathrm{C}_{26} \mathrm{H}_{42} \mathrm{O}_{4} \mathrm{Si}+\mathrm{Na}\right]^{+}$469.2750, found 469.2755 .

\section{General Procedure for TMSOTf-Mediated Cyclizations:}

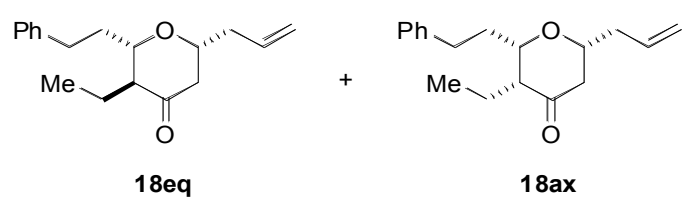

6-Allyl-3-ethyl-2-phenethyltetrahydropyran-4-one

(18).

Trimethylsilyl

trifluoromethanesulfonate $\left(36 \mu \mathrm{L}, 201 \mu \mathrm{mol}, 3.0\right.$ equiv.) was added to a cooled $\left(-78{ }^{\circ} \mathrm{C}\right)$ solution of $\alpha$-acetoxy ether 11 (30 mg, $67 \mu \mathrm{mol}, 1.0$ equiv.) in $\mathrm{CH}_{2} \mathrm{Cl}_{2}(1.3 \mathrm{~mL})$. After 30 min. of stirring at $-78{ }^{\circ} \mathrm{C}$, no starting acetoxy ether remained and the reaction mixture was quenched with satd. aq. $\mathrm{NaHCO}_{3}(2 \mathrm{~mL})$. The aqueous was extracted with diethyl ether $(3 \times 10 \mathrm{~mL})$, washed with brine $(10 \mathrm{~mL})$, dried $\left(\mathrm{MgSO}_{4}\right)$ and concentrated. Purification $\left(\mathrm{SiO}_{2}, 5 \rightarrow 10 \%\right.$ ether/hexanes, $\left.\mathrm{R}_{\mathrm{f}}=0.20\right)$ provided $11 \mathrm{mg}(61 \%)$ of $\mathbf{1 8 e q}$ and $7 \mathrm{mg}(38 \%)$ of 18ax.

C-3 equatorial product 18eq (confirmed by NOESY studies): IR (neat) 2927, 1715, 1456, $1111 \mathrm{~cm}^{-1} ;{ }^{1} \mathrm{H}$ NMR (400 MHz, $\left.\mathrm{CDCl}_{3}\right) \delta$ 7.31-7.29 (m, $\left.2 \mathrm{H}\right), 7.22-7.17$ (m, $\left.3 \mathrm{H}\right), 5.89$ (dddd, $J=17.0,10.1,7.1,7.1 \mathrm{~Hz}, 1 \mathrm{H}), 5.18-5.11(\mathrm{~m}, 2 \mathrm{H}), 3.64-3.56(\mathrm{~m}, 1 \mathrm{H}), 3.31$ (ddd, $J=9.8,9.8,2.4 \mathrm{~Hz}, 1 \mathrm{H}), 2.96-2.88(\mathrm{~m}, 1 \mathrm{H}), 2.77-2.68(\mathrm{~m}, 1 \mathrm{H}), 2.49-2.28(\mathrm{~m}, 4$ H), 2.20-2.14 (m, $1 \mathrm{H}), 2.06-1.97(\mathrm{~m}, 1 \mathrm{H}), 1.91-1.81(\mathrm{~m}, 1 \mathrm{H}), 0.82$ (t, $J=7.4 \mathrm{~Hz}, 3 \mathrm{H})$; ${ }^{13} \mathrm{C}$ NMR $\left(125 \mathrm{MHz}, \mathrm{CDCl}_{3}\right) \delta 208.4,141.9,133.8,128.5,128.4,125.9,117.7,79.1$, 76.6, 56.4, 47.9, 40.7, 35.8, 31.6, 17.1, 11.6. Anal. Calcd for $\mathrm{C}_{18} \mathrm{H}_{24} \mathrm{O}_{2}$ : C, 79.37; $\mathrm{H}$, 8.88. Found: C, 79.50; H, 9.05 . 
C-3 axial product 18ax: IR (neat) 2926, 1717, 1456, $1095 \mathrm{~cm}^{-1} ;{ }^{1} \mathrm{H}$ NMR $(500 \mathrm{MHz}$, $\left.\mathrm{C}_{6} \mathrm{D}_{6}\right) \delta$ 7.18-7.15 (m, $\left.2 \mathrm{H}\right), 7.12-7.04$ (m, $\left.3 \mathrm{H}\right), 5.77$ (dddd, $J=17.1,10.2,7.0,7.0 \mathrm{~Hz}, 1$ H), 5.04-4.95 (m, 2 H), 3.24-3.16 (m, 2 H), 2.68 (ddd, $J=13.7,9.8,5.2 \mathrm{~Hz}, 1 \mathrm{H}), 2.45$ (dddd, $J=9.3,6.8,6.8$,6.8 Hz, $1 \mathrm{H}), 2.19-2.13$ (m, $1 \mathrm{H}), 2.08-1.98$ (m, $3 \mathrm{H}), 190-1.82$ $(\mathrm{m}, 1 \mathrm{H}), 1.53-1.28(\mathrm{~m}, 4 \mathrm{H}), 0.75(\mathrm{t}, J=7.6 \mathrm{~Hz}, 3 \mathrm{H}) ;{ }^{13} \mathrm{C}$ NMR $\left(125 \mathrm{MHz}, \mathrm{C}_{6} \mathrm{D}_{6}\right) \delta$ 208.6, 142.4, 134.6, 129.1, 129.1, 128.7, 128.7, 126.6, 117.9, 79.1, 77.3, 57.3, 44.9, 41.2, 34.5, 33.0, 18.7, 12.0. Anal. Calcd for $\mathrm{C}_{18} \mathrm{H}_{24} \mathrm{O}_{2}$ : C, 79.37; H, 8.88. Found: C, 79.50; H, 8.96.

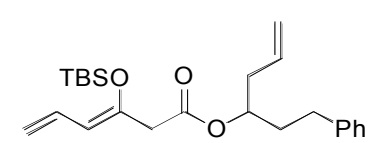

3-(tert-Butyldimethylsilanyloxy)-hexa-3,5-dienoic acid 1-phenethylbut-3-enyl ester (104). A sample of $369 \mathrm{mg}$ of 8 was converted to $425 \mathrm{mg} \mathrm{(80 \% )}$ of 104, isolated as the Z-alkene (confirmed by NOE studies): IR (neat) 2931, 1737, 1751, 1421, 1002, 840, 699 $\mathrm{cm}^{-1} ;{ }^{1} \mathrm{H}$ NMR (400 MHz, $\mathrm{C}_{6} \mathrm{D}_{6}$ ) $\delta$ 7.19-7.13 (m, $\left.2 \mathrm{H}\right), 7.10-7.04$ (m, $\left.3 \mathrm{H}\right), 6.85$ (ddd, $J$ $=17.2,10.5,10.5 \mathrm{~Hz}, 1 \mathrm{H}), 5.68(\mathrm{dddd}, J=17.9,9.3,7.1,7.1 \mathrm{~Hz}, 1 \mathrm{H}), 5.43(\mathrm{~d}, J=10.7$ Hz, $1 \mathrm{H}), 5.09-4.90$ (m, 5 H), 2.95 (s, $2 \mathrm{H}), 2.65-2.45$ (m, $2 \mathrm{H}), 2.25-2.12(\mathrm{~m}, 2 \mathrm{H})$, 1.85-1.61 (m, $2 \mathrm{H}), 0.96$ (s, $9 \mathrm{H}), 0.14$ (s, $3 \mathrm{H}), 0.13$ (s, $3 \mathrm{H}) ;{ }^{13} \mathrm{C}$ NMR (100 MHz, $\left.\mathrm{C}_{6} \mathrm{D}_{6}\right)$ $\delta 169.3,146.6,142.3,134.2,131.9,129.1,126.6,118.2,114.5,114.0,73.7,43.7,39.3$, 36.1, 32.4, 26.2, 18.8, -3.6; HRMS (EI) calcd for $\left[\mathrm{C}_{24} \mathrm{H}_{36} \mathrm{O}_{3} \mathrm{Si}+\mathrm{Na}\right]^{+}$423.2332, found 423.2333 .

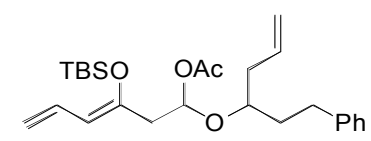

Acetic acid 3-(tert-butyldimethylsilanyloxy)-1-(1-phenethylbut-3-enyloxy)hexa-3,5dienyl ester (12). A sample of $231 \mathrm{mg}$ of $\mathbf{1 0 4}$ was converted to $250 \mathrm{mg}(92 \%)$ of $\mathbf{1 2}$, 
isolated as a 1:1 mixture of diastereomers: IR (neat) 2931, 1741, 1651, 1420, 1002, 839, $699 \mathrm{~cm}^{-1} ;{ }^{1} \mathrm{H}$ NMR $\left(500 \mathrm{MHz}, \mathrm{C}_{6} \mathrm{D}_{6}\right) \delta$ 7.23-7.04 (m, $\left.5 \mathrm{H}\right), 6.87-6.78(\mathrm{~m}, 1 \mathrm{H}), 6.45-$ $6.39(\mathrm{~m}, 1 \mathrm{H}), 5.92-5.77(\mathrm{~m}, 1 \mathrm{H}), 5.47(\mathrm{~d}, J=10.7 \mathrm{~Hz}, 0.5 \mathrm{H}), 5.46(\mathrm{~d}, J=10.7 \mathrm{~Hz}, 0.5$ H), 5.10-4.99 (m, $3 \mathrm{H}), 4.99-4.89(\mathrm{~m}, 1 \mathrm{H}), 3.79-3.70$ (m, $1 \mathrm{H}), 2.86-2.70(\mathrm{~m}, 1 \mathrm{H})$, 2.66-2.53 (m, 3 H), 2.36-2.20 (m, 2 H), 1.87-1.69 (m, 2 H), 1.70 (s, 3 H), 1.00 (s, 4.5 H), 0.98 (s, $4.5 \mathrm{H}), 0.16(\mathrm{~s}, 3 \mathrm{H}), 0.13(\mathrm{~s}, 1.5 \mathrm{H}), 0.12$ (s, $1.5 \mathrm{H}) ;{ }^{13} \mathrm{C}$ NMR $(125 \mathrm{MHz}$, $\left.\mathrm{C}_{6} \mathrm{D}_{6}\right) \delta 170.5,170.4,148.2,148.2,143.0,142.9,135.4,134.7,132.1,129.2,129.1$, $129.0,129.0,128.7,126.5,118.1,117.3,114.7,114.5,113.7,113.6,96.7,95.2,79.7$, $77.9,43.7,43.5,40.2,39.2,36.9,36.8,32.5,31.9,26.3,21.3,21.2,18.8,-3.4,-3.5$; HRMS (EI) calcd for $\left[\mathrm{C}_{26} \mathrm{H}_{40} \mathrm{O}_{4} \mathrm{Si}+\mathrm{Na}\right]^{+} 467.2594$, found 467.2604.

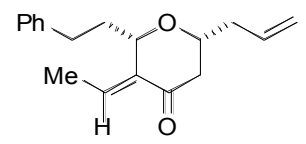

6-Allyl-3-ethylidene-2-phenethyltetrahydropyran-4-one (105). A sample of $70 \mathrm{mg}$ of 12 was converted to $35 \mathrm{mg}$ (84\%) of 19. Proton NMR analysis of the crude mixture allowed a 2:1 ratio of equatorial and axial products $\mathbf{1 9}$ to be assigned. However, upon attempted separation on triethylamine-deactivated silica gel produced $\mathbf{1 0 5}$, isolated as a single $E$-ethylidene isomer (NOE): IR (neat) 2925, 1696, 1622, $1091 \mathrm{~cm}^{-1} ;{ }^{1} \mathrm{H}$ NMR (500 MHz, $\left.\mathrm{C}_{6} \mathrm{D}_{6}\right) \delta$ 7.18-7.14 (m, $\left.2 \mathrm{H}\right), 7.12-7.05(\mathrm{~m}, 3 \mathrm{H}), 6.64(\mathrm{dq}, J=7.3,2.1 \mathrm{~Hz}, 1 \mathrm{H})$, 5.77 (dddd, $J=17.0,10.3,7.1,7.1 \mathrm{~Hz}, 1 \mathrm{H}), 5.06-4.98(\mathrm{~m}, 2 \mathrm{H}), 4.49-4.45(\mathrm{~m}, 1 \mathrm{H})$, 3.38-3.32 (m, $1 \mathrm{H}), 2.81-2.69$ (m, $2 \mathrm{H}), 2.20-2.12(\mathrm{~m}, 3 \mathrm{H}), 2.00-1.93(\mathrm{~m}, 1 \mathrm{H}), 1.82-$ $1.73(\mathrm{~m}, 2 \mathrm{H}), 1.16(\mathrm{~d}, J=7.5 \mathrm{~Hz}, 3 \mathrm{H}) ;{ }^{13} \mathrm{C} \mathrm{NMR}\left(125 \mathrm{MHz}, \mathrm{C}_{6} \mathrm{D}_{6}\right) \delta 196.8,142.6$, $140.1,134.7,129.3,129.0,128.7,128.3,126.5,117.7,74.5,72.5,45.4,40.5,38.3,31.8$, 14.1; HRMS (EI) calcd for $\left[\mathrm{C}_{18} \mathrm{H}_{22} \mathrm{O}_{2}+\mathrm{Na}\right]^{+} 293.1518$, found 293.1505 . 


\section{General Procedure for the Synthesis of Silyl Enol Ethers (Method B):}

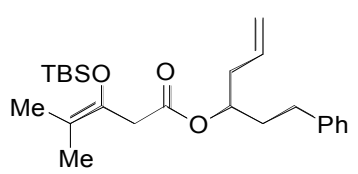

3-(tert-Butyldimethylsilanyloxy)-4-methyl-pent-3-enoic acid 1-phenethylbut-3-enyl ester (106). To a cooled $\left(0{ }^{\circ} \mathrm{C}\right)$ suspension of zinc dust (118 mg, $1.80 \mathrm{mmol}, 6.0$ equiv.) in THF was added $\alpha$-bromoisobutyryl bromide $(0.11 \mathrm{~mL}, 0.90 \mathrm{mmol}, 3.0$ equiv.). After $30 \mathrm{~min}$, the green solution was transferred via cannula to a $0{ }^{\circ} \mathrm{C}$ solution of silyl ketene acetal 8 (80 $\mathrm{mg}, 0.24 \mathrm{mmol}, 1.0$ equiv.) in THF. The reaction mixture was stirred at room temperature for $16 \mathrm{~h}$. The reaction mixture was diluted with diethyl ether, washed with $\mathrm{H}_{2} \mathrm{O}$ then satd. aq. $\mathrm{NaCl}$ and dried over anhydrous $\mathrm{MgSO}_{4}$. After concentration and purification ( $10 \%$ diethyl ether/hexanes, $\left.\mathrm{R}_{\mathrm{f}}=0.38\right), 70 \mathrm{mg}(72 \%)$ of 106 was isolated as a colorless oil: IR (neat) 2930, 1737, 1455, 1256, 1197, $836 \mathrm{~cm}^{-1} ;{ }^{1} \mathrm{H}$ NMR (500 MHz, $\left.\mathrm{C}_{6} \mathrm{D}_{6}\right) \delta 7.18-7.14(\mathrm{~m}, 2 \mathrm{H}), 7.10-7.04(\mathrm{~m}, 3 \mathrm{H}), 5.72-5.63(\mathrm{~m}, 1 \mathrm{H}), 5.05-4.95(\mathrm{~m}, 3 \mathrm{H})$, $3.14(\mathrm{~d}, J=15.7 \mathrm{~Hz}, 1 \mathrm{H}), 3.10(\mathrm{~d}, J=15.7 \mathrm{~Hz}, 1 \mathrm{H}), 2.65-2.58(\mathrm{~m}, 1 \mathrm{H}), 2.54-2.46(\mathrm{~m}$, $1 \mathrm{H}), 2.24-2.14$ (m, $2 \mathrm{H}), 1.84-1.75(\mathrm{~m}, 1 \mathrm{H}), 1.71-1.65(\mathrm{~m}, 1 \mathrm{H}), 1.73$ (s, $3 \mathrm{H}), 1.59$ (s, $3 \mathrm{H}), 1.01(\mathrm{~s}, 9 \mathrm{H}), 0.18(\mathrm{~s}, 3 \mathrm{H}), 0.17(\mathrm{~s}, 3 \mathrm{H}) ;{ }^{13} \mathrm{C} \mathrm{NMR}\left(125 \mathrm{MHz}, \mathrm{C}_{6} \mathrm{D}_{6}\right) \delta 170.1$, $142.3,138.7,134.4,129.1,129.1,128.7,126.6,118.0,113.5,73.3,39.6,39.4,36.2,32.4$, 26.5, 19.6, 18.8, 18.6, -3.4, -3.4; HRMS (EI) calcd for $\left[\mathrm{C}_{24} \mathrm{H}_{38} \mathrm{O}_{3} \mathrm{Si}+\mathrm{H}\right]^{+}$403.2668, found 403.2666 .

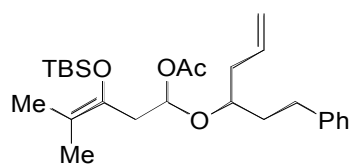


Acetic acid 3-(tert-butyldimethylsilanyloxy)-4-methyl-1-(1-phenethylbut-3enyloxy)pent-3-enyl ester (13). A sample of $300 \mathrm{mg}$ of $\mathbf{1 0 6}$ was converted to $306 \mathrm{mg}$ $(92 \%)$ of 13 , isolated as 1:1 mixture of diastereomers: IR (neat) 2930, 1741, 1682, 1124, 1007, 838, $700 \mathrm{~cm}^{-1} ;{ }^{1} \mathrm{H}$ NMR (500 MHz, $\left.\mathrm{C}_{6} \mathrm{D}_{6}\right) \delta$ 7.23-7.21 (m, $\left.2 \mathrm{H}\right), 7.16-7.04(\mathrm{~m}, 3$ H), 6.45-6.41 (m, $1 \mathrm{H})$, 5.92-5.79 (m, $1 \mathrm{H}), 5.07-4.99(\mathrm{~m}, 2 \mathrm{H}), 3.79-3.73(\mathrm{~m}, 1 \mathrm{H})$, 2.87-2.71 (m, $2 \mathrm{H}), 2.65-2.53(\mathrm{~m}, 2 \mathrm{H}), 2.38-2.21(\mathrm{~m}, 2 \mathrm{H}), 1.87-1.74(\mathrm{~m}, 2 \mathrm{H}), 1.72$ (s, $1.5 \mathrm{H}), 1.72(\mathrm{~s}, 1.5 \mathrm{H}), 1.70(\mathrm{~s}, 1.5 \mathrm{H}), 1.68(\mathrm{~s}, 1.5 \mathrm{H}), 1.68(\mathrm{~s}, 1.5 \mathrm{H}), 1.67(\mathrm{~s}, 1.5 \mathrm{H})$, $1.04(\mathrm{~s}, 9 \mathrm{H}), 0.18(\mathrm{~s}, 1.5 \mathrm{H}), 0.17(\mathrm{~s}, 1.5 \mathrm{H}), 0.13(\mathrm{~s}, 1.5 \mathrm{H}), 0.12(\mathrm{~s}, 1.5) ;{ }^{13} \mathrm{C} \mathrm{NMR}(125$ $\left.\mathrm{MHz}, \mathrm{C}_{6} \mathrm{D}_{6}\right) \delta 170.5,170.3,143.1,143.0,140.2,140.1,135.5,134.8,129.2,129.1,129.1$, $129.0,128.7,128.2,126.5,126.4,117.8,117.3,113.6,113.4,97.6,96.2,79.5,77.9,40.3$, $39.2,39.1,38.9,37.0,36.9,32.5,31.6,26.5,26.4,21.3,21.3,19.7,19.6,18.8,18.8,18.6$, $-3.2,-3.2,-3.4,-3.4$; HRMS (EI) calcd for $\left[\mathrm{C}_{26} \mathrm{H}_{42} \mathrm{O}_{4} \mathrm{Si}+\mathrm{Na}\right]^{+} 469.2750$, found 469.2752 .

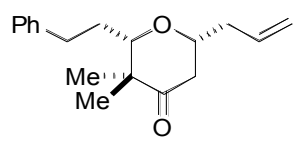

6-Allyl-3,3-dimethyl-2-phenethyltetrahydropyran-4-one (20). A sample of $40 \mathrm{mg}$ of $\mathbf{1 3}$ was converted to $22 \mathrm{mg}(92 \%)$ of $\mathbf{2 0}$, isolated as a colorless oil: IR (neat) 2932, 1712, 1455, 1127, $700 \mathrm{~cm}^{-1} ;{ }^{1} \mathrm{H}$ NMR (500 MHz, $\left.\mathrm{C}_{6} \mathrm{D}_{6}\right) \delta$ 7.19-7.15 (m, $\left.2 \mathrm{H}\right), 7.11-7.07$ (m, 3 H), 5.84-5.74 (m, 1 H), 5.06-4.95 (m, $2 \mathrm{H}), 3.22-3.14(\mathrm{~m}, 1 \mathrm{H}), 2.95(\mathrm{~d}, J=10.6 \mathrm{~Hz}, 1$ H), 2.912-2.84 (m, $1 \mathrm{H}), 2.60-2.52(\mathrm{~m}, 1 \mathrm{H}), 2.23-2.13(\mathrm{~m}, 2 \mathrm{H}), 2.07-1.95(\mathrm{~m}, 2 \mathrm{H})$, $1.77-1.68(\mathrm{~m}, 1 \mathrm{H}), 1.51-1.43(\mathrm{~m}, 1 \mathrm{H}), 0.92(\mathrm{~s}, 3 \mathrm{H}), 0.90(\mathrm{~s}, 3 \mathrm{H}) ;{ }^{13} \mathrm{C} \mathrm{NMR}(125 \mathrm{MHz}$, $\left.\mathrm{C}_{6} \mathrm{D}_{6}\right) \delta 209.9,142.7,134.6,129.2,129.1,128.7,128.2,128.6,117.9,83.4,77.1,49.4$, 
44.4, 41.2, 33.4, 31.7, 19.5, 19.3; HRMS (EI) calcd for $\left[\mathrm{C}_{18} \mathrm{H}_{24} \mathrm{O}_{2}+\mathrm{Na}\right]^{+}$295.1674, found 295.1669. Anal. Calcd for $\mathrm{C}_{18} \mathrm{H}_{24} \mathrm{O}_{2}$ : C, 79.37; H, 8.88. Found: C, 79.50; H, 9.00.

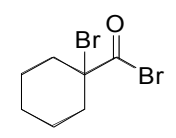

1-Bromocyclohexanecarbonyl bromide (107). Known compound: ${ }^{3}$ IR (neat) 2941, 1770, 1081, 956, $627 \mathrm{~cm}^{-1} ;{ }^{1} \mathrm{H}$ NMR (400 MHz, $\left.\mathrm{C}_{6} \mathrm{D}_{6}\right) \delta$ 1.90-1.70 (m, $\left.4 \mathrm{H}\right), 1.41-1.28$ (m, $2 \mathrm{H}), 1.22-1.10(\mathrm{~m}, 2 \mathrm{H}), 1.08-0.96(\mathrm{~m}, 1 \mathrm{H}), 0.92-0.79$ (m, $1 \mathrm{H}) ;{ }^{13} \mathrm{C}$ NMR $(100$ $\left.\mathrm{MHz}, \mathrm{C}_{6} \mathrm{D}_{6}\right) \delta 170.7,73.6,39.0,24.8,23.9$.

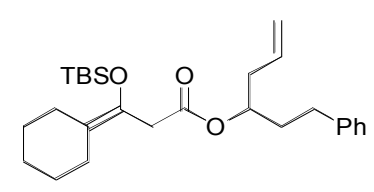

3-(tert-Butyldimethylsilanyloxy)-3-cyclohexylidene-propionic acid 1-phenethylbut-3enyl ester (108). A sample of $40 \mathrm{mg}$ of 8 was converted to $36 \mathrm{mg}(68 \%)$ of $\mathbf{1 0 8}$, isolated as a colorless oil: IR (neat) 2929, 1737, 1450, 1032, $838 \mathrm{~cm}^{-1} ;{ }^{1} \mathrm{H}$ NMR $(500 \mathrm{MHz}$, $\left.\mathrm{C}_{6} \mathrm{D}_{6}\right) \delta$ 7.20-7.05 (m, $\left.5 \mathrm{H}\right), 5.76-5.65(\mathrm{~m}, 1 \mathrm{H}), 5.07-5.02(\mathrm{~m}, 1 \mathrm{H}), 5.01-4.96(\mathrm{~m}, 2 \mathrm{H})$, $3.17(\mathrm{~d}, J=15.5 \mathrm{~Hz}, 1 \mathrm{H}), 3.13(\mathrm{~d}, J=15.5 \mathrm{~Hz}, 1 \mathrm{H}), 2.68-2.60(\mathrm{~m}, 1 \mathrm{H}), 2.56-2.48(\mathrm{~m}$, $1 \mathrm{H}), 2.35-2.28$ (m, $2 \mathrm{H}), 2.24-2.16(\mathrm{~m}, 2 \mathrm{H}), 2.12-2.06(\mathrm{~m}, 2 \mathrm{H}), 1.87-1.78(\mathrm{~m}, 1 \mathrm{H})$, 1.74-1.64 (m, $1 \mathrm{H}), 1.57-1.41(\mathrm{~m}, 6 \mathrm{H}), 1.02(\mathrm{~s}, 9 \mathrm{H}), 0.19(\mathrm{~s}, 3 \mathrm{H}), 0.19(\mathrm{~s}, 3 \mathrm{H}) ;{ }^{13} \mathrm{C}$ NMR (125 MHz, $\left.\mathrm{C}_{6} \mathrm{D}_{6}\right) \delta$ 170.2, 142.4, 136.0, 134.4, 129.1, 128.7, 126.6, 121.8, 118.0, $73.3,39.4,39.2,36.3,32.4,30.6,28.5,28.2,27.9,27.4,26.7,26.5,18.9,-3.5,-3.5$; HRMS (EI) calcd for $\left[\mathrm{C}_{27} \mathrm{H}_{42} \mathrm{O}_{3} \mathrm{Si}+\mathrm{H}\right]^{+}$443.2982, found 443.2974.

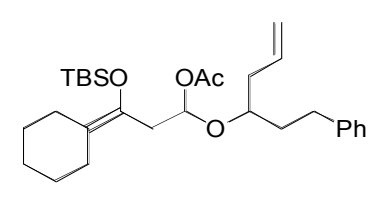

\footnotetext{
${ }^{3}$ Wagner, R. B.; Moore, J. A. J. Am. Chem. Soc. 1950, 72, 974-977.
} 
Acetic acid 3-(tert-butyldimethylsilanyloxy)-3-cyclohexylidene-1-(1-phenethylbut-3enyloxy)propyl ester (14). A sample of $169 \mathrm{mg}$ of 108 was converted to $172 \mathrm{mg}(93 \%)$ of 14, isolated as 1:1 mixture of diastereomers: IR (neat) 2928, 1739, 1448, 1250, 1007, $838 \mathrm{~cm}^{-1} ;{ }^{1} \mathrm{H}$ NMR $\left(500 \mathrm{MHz}, \mathrm{C}_{6} \mathrm{D}_{6}\right) \delta 7.24-7.14(\mathrm{~m}, 5 \mathrm{H}), 7.11-7.04(\mathrm{~m}, 1 \mathrm{H}), 6.47-$ $6.42(\mathrm{~m}, 1 \mathrm{H})$, 5.95-5.85 (m, $1 \mathrm{H})$, 5.10-5.00 (m, $2 \mathrm{H}), 3.81-3.73$ (m, $1 \mathrm{H}), 2.90-2.75$ (m, $2 \mathrm{H}), 2.72-2.57(\mathrm{~m}, 2 \mathrm{H}), 2.43-2.12(\mathrm{~m}, 6 \mathrm{H}), 1.91-1.82(\mathrm{~m}, 1 \mathrm{H}), 1.75(\mathrm{~s}, 1.5 \mathrm{H})$, $1.74(\mathrm{~s}, 1.5 \mathrm{H}), 1.62-1.42(\mathrm{~m}, 6 \mathrm{H}), 1.05(\mathrm{~s}, 4.5 \mathrm{H}), 1.04(\mathrm{~s}, 4.5 \mathrm{H}), 0.19$ (s, $1.5 \mathrm{H}), 0.18$ $(\mathrm{s}, 1.5 \mathrm{H}), 0.15(\mathrm{~s}, 1.5 \mathrm{H}), 0.15(\mathrm{~s}, 1.5 \mathrm{H}) ;{ }^{13} \mathrm{C} \mathrm{NMR}\left(125 \mathrm{MHz}, \mathrm{C}_{6} \mathrm{D}_{6}\right) \delta$ 170.2, 170.2, $143.0,137.4,135.6,135.0,129.2,129.1,129.0,128.7,126.5,126.4,121.8,117.8,117.3$, $97.5,96.1,79.3,77.8,40.3,39.3,38.6,38.5,37.0,36.9,32.5,31.8,30.5,28.8,28.7,28.3$, 28.0, 27.9, 27.5, 27.5, 26.5, 26.5, 21.4, 21.3, 18.8, -3.2, -3.5; HRMS (EI) calcd for $\left[\mathrm{C}_{29} \mathrm{H}_{46} \mathrm{O}_{4} \mathrm{Si}+\mathrm{Na}\right]^{+}$509.3063, found 509.3063.

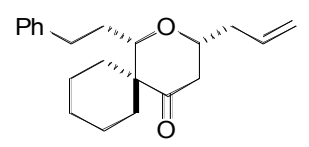

3-Allyl-1-phenethyl-2-oxaspiro[5.5] undecan-5-one (21). A sample of $43 \mathrm{mg}$ of $\mathbf{1 4}$ was converted to $26 \mathrm{mg}(93 \%)$ of $\mathbf{2 1}$, isolated as a colorless oil: IR (neat) 2930, 1706, 1455, 1239, $1120 \mathrm{~cm}^{-1} ;{ }^{1} \mathrm{H}$ NMR (500 MHz, $\left.\mathrm{C}_{6} \mathrm{D}_{6}\right) \delta 7.20-7.15(\mathrm{~m}, 2 \mathrm{H}), 7.12-7.07(\mathrm{~m}, 3 \mathrm{H})$, 5.79 (dddd, $J=17.1,10.2,7.0,7.0 \mathrm{~Hz}, 1 \mathrm{H}), 5.05-4.97(\mathrm{~m}, 2 \mathrm{H}), 3.30-3.23(\mathrm{~m}, 1 \mathrm{H})$, 2.94-2.86 (m, $2 \mathrm{H}), 2.50(\mathrm{ddd}, J=13.5,9.4,7.2 \mathrm{~Hz}, 1 \mathrm{H}), 2.36(\mathrm{dd}, J=12.3,12.3 \mathrm{~Hz}, 1$ H), 2.27-2.16 (m, $2 \mathrm{H}), 2.07-1.99(\mathrm{~m}, 3 \mathrm{H}), 1.84-1.70(\mathrm{~m}, 2 \mathrm{H}), 1.64-1.50(\mathrm{~m}, 3 \mathrm{H})$, $1.45-1.38(\mathrm{~m}, 1 \mathrm{H}), 1.25(\mathrm{ddd}, J=13.4,13.4,3.2 \mathrm{~Hz}, 1 \mathrm{H}), 1.02-0.88(\mathrm{~m}, 2 \mathrm{H}), 0.85$ (ddd, $J=12.8,12.8,4.7 \mathrm{~Hz}, 1 \mathrm{H}) ;{ }^{13} \mathrm{C} \mathrm{NMR}\left(125 \mathrm{MHz}, \mathrm{C}_{6} \mathrm{D}_{6}\right) \delta 211.3,142.8,134.7$, $129.2,129.1,128.7,126.6,117.9,85.2,78.5,55.1,45.7,41.2,33.9,31.9,29.2,28.6,27.2$, 
23.5, 23.1; HRMS (EI) calcd for $\left[\mathrm{C}_{21} \mathrm{H}_{28} \mathrm{O}_{2}+\mathrm{Na}\right]^{+}$335.1987, found 335.1993. Anal. Calcd for $\mathrm{C}_{21} \mathrm{H}_{28} \mathrm{O}_{2}$ : C, 80.73; H 9.03. Found: C, 80.61; H, 9.07.

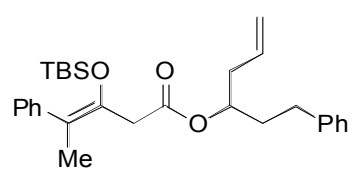

3-(tert-Butyldimethylsilanyloxy)-4-phenyl-pent-3-enoic acid 1-phenethylbut-3-enyl ester (109). A sample of $100 \mathrm{mg}$ of 8 was converted to $129 \mathrm{mg}(92 \%)$ of $\mathbf{1 0 9}$, isolated as a single $Z$-isomer (NOE): IR (neat) 2929, 1738, 1455, 1254, 829, $699 \mathrm{~cm}^{-1} ;{ }^{1} \mathrm{H}$ NMR (500 MHz, $\left.\mathrm{C}_{6} \mathrm{D}_{6}\right) \delta$ 7.40-7.36 (m, $\left.2 \mathrm{H}\right), 7.20-7.11(\mathrm{~m}, 6 \mathrm{H}), 7.09-7.01(\mathrm{~m}, 2 \mathrm{H}), 5.74$ (dddd, $J=17.7,9.6,7.0,7.0 \mathrm{~Hz}, 1 \mathrm{H}), 5.13-5.07$ (m, $1 \mathrm{H}), 5.04-4.99$ (m, $2 \mathrm{H}), 3.25(\mathrm{~d}, J$ $=15.5 \mathrm{~Hz}, 1 \mathrm{H}), 3.21(\mathrm{~d}, J=15.5 \mathrm{~Hz}, 1 \mathrm{H}), 2.74-2.66(\mathrm{~m}, 1 \mathrm{H}), 2.61-2.53(\mathrm{~m}, 1 \mathrm{H})$, 2.28-2.19 (m, $2 \mathrm{H}), 1.92(\mathrm{~s}, 3 \mathrm{H}), 1.89-1.81(\mathrm{~m}, 1 \mathrm{H}), 1.74-1.66(\mathrm{~m}, 1 \mathrm{H}), 0.85(\mathrm{~s}, 9 \mathrm{H})$, $-0.11(\mathrm{~s}, 3 \mathrm{H}),-0.14(\mathrm{~s}, 3 \mathrm{H}) ;{ }^{13} \mathrm{C} \mathrm{NMR}\left(125 \mathrm{MHz}, \mathrm{C}_{6} \mathrm{D}_{6}\right) \delta$ 169.7, 412.6, 142.2, 140.7, $134.4,134.4,129.9,129.1,129.1,128.6,126.8,126.7,118.7,118.2,73.5,40.6,39.4$, $36.2,32.4,26.3,26.3,20.0,18.6,-3.9,-3.9$; HRMS (EI) calcd for $\left[\mathrm{C}_{29} \mathrm{H}_{40} \mathrm{O}_{3} \mathrm{Si}+\mathrm{Na}\right]^{+}$ 487.2644, found 487.2644 .

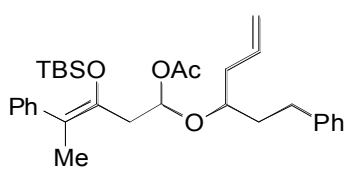

Acetic acid 3-(tert-butyldimethylsilanyloxy)-1-(1-phenethylbut-3-enyloxy)-4-phenylpent-3-enyl ester (15). A sample of $102 \mathrm{mg}$ of 109 was converted to $110 \mathrm{mg}(99 \%)$ of 15, isolated as a 1:1 mixture of diastereomers: IR (neat) 2930, 1741, 1455, 1007, 837, $700 \mathrm{~cm}^{-1} ;{ }^{1} \mathrm{H}$ NMR $\left(500 \mathrm{MHz}, \mathrm{C}_{6} \mathrm{D}_{6}\right) \delta 7.38-7.34(\mathrm{~m}, 1 \mathrm{H}), 7.33-7.29(\mathrm{~m}, 1 \mathrm{H}), 7.21-$ $7.11(\mathrm{~m}, 6 \mathrm{H}), 7.10-6.99$ (m, $2 \mathrm{H}), 6.63-6.58$ (m, $1 \mathrm{H})$, 5.98-5.86 (m, 1 H), 5.11-5.02 (m, 2 H), 3.86-3.77 (m, 1 H), 2.90-2.62 (m, 4 H), 2.41-2.27 (m, 2 H), 2.01-1.92 (m, 1 
H), 1.98 (s, 3 H), 1.90-1.80 (m, 1 H), 1.76 (s, 1.5 H), 1.76 (s, 1.5 H), 0.88 (s, 4.5 H), 0.87 $(\mathrm{s}, 4.5 \mathrm{H}),-0.14(\mathrm{~s}, 1.5 \mathrm{H}),-0.15(\mathrm{~s}, 1.5 \mathrm{H}),-0.17(\mathrm{~s}, 1.5 \mathrm{H}),-0.21(\mathrm{~s}, 1.5 \mathrm{H}) ;{ }^{13} \mathrm{C} \mathrm{NMR}$ $\left(125 \mathrm{MHz}, \mathrm{C}_{6} \mathrm{D}_{6}\right) \delta 170.4,170.3,143.0,142.9,142.7,142.0,135.4,134.8,129.9,129.1$, 129.1, 129.0, 128.7, 128.7, 128.6, 128.5, 126.7, 126.7, 126.5, 126.5, 118.9, 118.8, 118.1, $117.4,97.3,96.1,79.5,77.9,40.2,40.0,39.8,39.3,36.9,36.8,32.5,32.2,26.3,21.3$, $21.3,20.0,20.0,18.6,-3.8,-3.8,-3.9,-3.9$; HRMS (EI) calcd for $\left[\mathrm{C}_{31} \mathrm{H}_{44} \mathrm{O}_{4} \mathrm{Si}+\mathrm{Na}\right]^{+}$ 531.2906, found 531.2908.

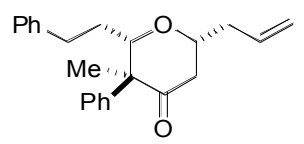

6-Allyl-3-methyl-2-phenethyl-3-phenyltetrahydropyran-4-one (22). A sample of 26 $\mathrm{mg}$ of $\mathbf{1 5}$ was converted to $15 \mathrm{mg}(88 \%)$ of $\mathbf{2 2}$, isolated as single diastereomer (confirmed by NOESY studies): IR (neat) 2927, 1713, 1445, $1098 \mathrm{~cm}^{-1} ;{ }^{1} \mathrm{H}$ NMR (500 MHz, $\mathrm{CDCl}_{3}$ )

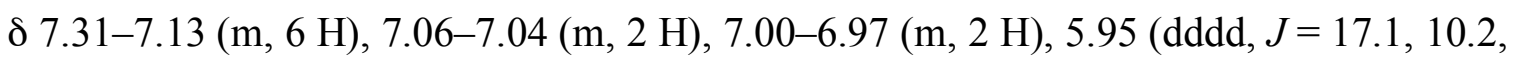
7.0, 7.0 Hz, $1 \mathrm{H}), 5.23-5.16$ (m, $2 \mathrm{H}), 3.88-3.83$ (m, $1 \mathrm{H}), 3.82$ (dd, $J=10.3,1.5 \mathrm{~Hz}, 1$ H), 2.86-2.80 (m, $1 \mathrm{H}), 2.66(\mathrm{dd}, J=15.5,11.8 \mathrm{~Hz}, 1 \mathrm{H}), 2.55-2.48(\mathrm{~m}, 2 \mathrm{H}), 2.44(\mathrm{dd}, J$ $=15.5,3.0 \mathrm{~Hz}, 1 \mathrm{H}), 2.42-2.37(\mathrm{~m}, 1 \mathrm{H}), 1.86-1.77(\mathrm{~m}, 1 \mathrm{H}), 1.57(\mathrm{~s}, 3 \mathrm{H}), 1.32-1.25$ (m, $1 \mathrm{H}) ;{ }^{13} \mathrm{C} \mathrm{NMR}\left(125 \mathrm{MHz}, \mathrm{CDCl}_{3}\right) \delta 210.6,141.5,139.6,133.6,128.4,128.2,128.2$, $127.9,127.0,125.8,117.9,82.9,76.5,58.1,43.9,405,32.5,30.5,16.1$; HRMS (EI) calcd for $\left[\mathrm{C}_{23} \mathrm{H}_{26} \mathrm{O}_{2}+\mathrm{Na}\right]^{+}$357.1830, found 357.1819.

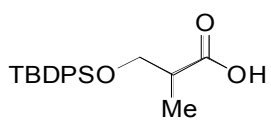


3-(tert-Butyldiphenylsilanyloxy)-2-methylpropionic acid (110). Known compound: ${ }^{4}$

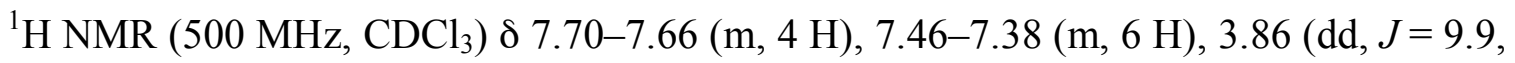
$6.9 \mathrm{~Hz}, 1 \mathrm{H}), 3.78(\mathrm{dd}, J=9.9,5.6 \mathrm{~Hz}, 1 \mathrm{H}), 2.79-2.72(\mathrm{~m}, 1 \mathrm{H}), 1.21(\mathrm{~d}, J=7.1 \mathrm{~Hz}, 3$ $\mathrm{H}), 1.09(\mathrm{~s}, 1 \mathrm{H}), 1.05(\mathrm{~s}, 9 \mathrm{H}) ;{ }^{13} \mathrm{C} \mathrm{NMR}\left(125 \mathrm{MHz}, \mathrm{CDCl}_{3}\right) \delta$ 190.9, 135.6, 135.6, $134.8,133.2,133.2,129.7,129.6,127.7,65.6,42.2,26.7,26.5,19.2,13.2$.

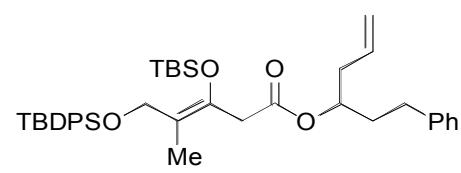

\section{3-(tert-Butyldimethylsilanyloxy)-5-(tert-butyldiphenylsilanyloxy)-4-methylpent-3-}

enoic acid 1-phenethylbut-3-enyl ester (111). A sample of $100 \mathrm{mg}$ of 8 was converted to $167 \mathrm{mg}(56 \%)$ of $\mathbf{1 1 1}$, isolated as a 1:2 E/Z mixture (crude product, 1:1.5 E/Z mixture): IR (neat) 2931, 1737, 1675, 1254, 1055, 834, $702 \mathrm{~cm}^{-1} ;{ }^{1} \mathrm{H}$ NMR (500 MHz, $\left.\mathrm{C}_{6} \mathrm{D}_{6}\right) \delta$ 7.85-7.80 (m, $4 \mathrm{H}), 7.26-7.03(\mathrm{~m}, 11 \mathrm{H}), 5.74-5.56(\mathrm{~m}, 1 \mathrm{H}), 5.07-4.89(\mathrm{~m}, 3 \mathrm{H}), 4.37$ $(\mathrm{s}, 0.7 \mathrm{H}), 3.14(\mathrm{~d}, J=16.3 \mathrm{~Hz}, 0.35 \mathrm{H}), 3.06(\mathrm{~d}, J=16.3 \mathrm{~Hz}, 0.35 \mathrm{H}), 3.04(\mathrm{~d}, J=15.9$ $\mathrm{Hz}, 0.15 \mathrm{H}), 3.00(\mathrm{~d}, J=15.9 \mathrm{~Hz}, 0.15 \mathrm{H}), 2.62$ (ddd, $J=13.4,10.3,5.3 \mathrm{~Hz}, 0.7 \mathrm{H})$, 2.58-2.48 (m, $1 \mathrm{H}), 2.46-2.39(\mathrm{~m}, 0.3 \mathrm{H}), 2.26-2.15(\mathrm{~m}, 1.4 \mathrm{H}), 2.14-2.09(\mathrm{~m}, 0.7 \mathrm{H})$, $1.95(\mathrm{~s}, 1 \mathrm{H}), 1.90(\mathrm{~s}, 2 \mathrm{H}), 1.87-1.79(\mathrm{~m}, 0.7 \mathrm{H}), 1.76-1.62(\mathrm{~m}, 1.3 \mathrm{H}), 1.20(\mathrm{~s}, 6 \mathrm{H})$, $1.18(\mathrm{~s}, 3 \mathrm{H}), 1.01(\mathrm{~s}, 3 \mathrm{H}), 0.85(\mathrm{~s}, 6 \mathrm{H}), 0.20(\mathrm{~d}, J=1.7 \mathrm{~Hz}, 2 \mathrm{H}), 0.06(\mathrm{~d}, J=2.7 \mathrm{~Hz}, 4$ $\mathrm{H}) ;{ }^{13} \mathrm{C}$ NMR $\left(125 \mathrm{MHz}, \mathrm{C}_{6} \mathrm{D}_{6}\right) \delta 169.8,169.7,142.3,141.6,139.8,136.5,136.4,134.7$, $134.7,134.3,134.2,130.4,130.2,129.1,129.1,128.7,128.6,128.3,126.6,126.6,118.1$, $118.1,117.7,73.6,73.5,65.6,63.0,39.8,39.4,39.4,39.3,36.2,36.1,32.4,32.3,27.6$, 27.5, 26.4, 26.3, 20.0, 19.9, 18.9, 18.7, 14.8, 14.8, -3.4, -3.7, -3.7; HRMS (EI) calcd for $\left[\mathrm{C}_{40} \mathrm{H}_{56} \mathrm{O}_{4} \mathrm{Si}_{2}+\mathrm{Na}\right]^{+}$679.3615, found 679.3585 .

\footnotetext{
${ }^{4}$ Heathcock, C. H.; Young, S. D.; Hagen, J. P.; Pilli, R.; Badertscher, U. J. Org. Chem. 1985, 50, 2095-2105.
} 


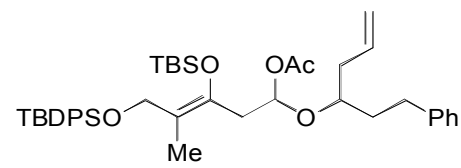

\section{Acetic acid 3-(tert-butyldimethylsilanyloxy)-5-(tert-butyldiphenylsilanyloxy)-4-} methyl-1-(1-phenethylbut-3-enyloxy)pent-3-enyl ester (16). A sample of $97 \mathrm{mg}$ of 111 was converted to $95 \mathrm{mg}(92 \%)$ of $\mathbf{1 6}$, isolated as a diastereomeric mixture of alkene isomers: IR (neat) 2931, 1741, 1674, 1237, 1049, 838, $701 \mathrm{~cm}^{-1} ;{ }^{1} \mathrm{H}$ NMR (500 MHz, $\left.\mathrm{C}_{6} \mathrm{D}_{6}\right) \delta$ 7.87-7.76 (m, $\left.4 \mathrm{H}\right), 7.30-7.05(\mathrm{~m}, 11 \mathrm{H}), 6.50-6.45(\mathrm{~m}, 0.8 \mathrm{H}), 6.46-6.32(\mathrm{~m}$, $0.2 \mathrm{H}), 5.94-5.74(\mathrm{~m}, 1 \mathrm{H}), 5.08-4.96(\mathrm{~m}, 2 \mathrm{H}), 4.57(\mathrm{~d}, J=8.1 \mathrm{~Hz}, 0.4 \mathrm{H}), 4.54(\mathrm{~d}, J=$ 8.1 Hz, 0.4 H), 4.50-4.37 (m, 1.2 H), 3.82-3.76 (m, 0.8 H), 3.72-3.68 (m, 0.2 H), 2.902.60 (m, 4 H), 2.38-2.16 (m, 2 H), 1.98 (s, $1 \mathrm{H}), 1.94-1.80$ (m, $2 \mathrm{H}), 1.76$ (s, $1.5 \mathrm{H}), 1.75$ $(\mathrm{s}, 1.5 \mathrm{H}), 1.67(\mathrm{~s}, 0.5 \mathrm{H}), 1.66(\mathrm{~s}, 0.5 \mathrm{H}), 1.22(\mathrm{~s}, 3 \mathrm{H}), 1.21(\mathrm{~s}, 2 \mathrm{H}), 1.20(\mathrm{~s}, 1 \mathrm{H}), 1.19$ (s, $3 \mathrm{H}), 1.05$ (s, $1 \mathrm{H}), 1.03(\mathrm{~s}, 1 \mathrm{H}), 0.89$ (s, $3.5 \mathrm{H}), 0.88(\mathrm{~s}, 3.5 \mathrm{H}), 0.20(\mathrm{~s}, 0.5 \mathrm{H}), 0.20$ $(\mathrm{s}, 0.5 \mathrm{H}), 0.17(\mathrm{~s}, 0.5 \mathrm{H}), 0.16(\mathrm{~s}, 0.5 \mathrm{H}), 0.04(\mathrm{~s}, 1 \mathrm{H}), 0.02(\mathrm{~s}, 1 \mathrm{H}), 0.02(\mathrm{~s}, 1 \mathrm{H}), 0.01$ $(\mathrm{s}, 1 \mathrm{H}) ;{ }^{13} \mathrm{C}$ NMR $\left(125 \mathrm{MHz}, \mathrm{C}_{6} \mathrm{D}_{6}\right) \delta 170.5,170.4,170.2,142.9,142.8,141.3,141.3$, $136.5,135.4,134.8,130.3,130.3,130.2,129.1,129.1,129.1,129.1,129.0,129.0,128.7$, $128.6,126.4,118.0,118.0,117.9,117.8,117.4,117.3,96.9,95.9,95.7,79.6,79.3,77.9$, $65.5,63.1,63.0,40.2,39.3,39.2,39.1,38.8,36.9,36.8,36.7,32.5,32.4,31.9,27,6,27.5$, $26.4,26.3,21.3,21.3,21.2,20.0,18.9,18.7,15.0,14.9,14.9,-3.1,-3.3,-3.5,-3.6,-3.6$; HRMS (EI) calcd for $\left[\mathrm{C}_{42} \mathrm{H}_{60} \mathrm{O}_{5} \mathrm{Si}_{2}+\mathrm{Na}\right]^{+} 723.3877$, found 723.3881 .

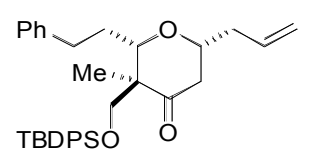

6-Allyl-3-(tert-butyldiphenylsilanyloxymethyl)-3-methyl-2-phenethyl-

tetrahydropyran-4-one (23). A sample of $26 \mathrm{mg}$ of 16 was converted to $15 \mathrm{mg}(77 \%)$ 
of $\mathbf{2 3}$, isolated as a 1:1.2 mixture of diastereomers: IR (neat) $2930,1717,1428,1112,702$ $\mathrm{cm}^{-1} ;{ }^{1} \mathrm{H}$ NMR $\left(500 \mathrm{MHz}, \mathrm{CDCl}_{3}\right) \delta$ 7.74-7.70 (m, $\left.1 \mathrm{H}\right), 7.62-7.55$ (m, $\left.2 \mathrm{H}\right), 7.45-7.09$ (m, $12 \mathrm{H}), 5.99-5.89(\mathrm{~m}, 0.7 \mathrm{H}), 5.84-5.75(\mathrm{~m}, 0.3 \mathrm{H}), 5.20-5.07(\mathrm{~m}, 2 \mathrm{H}), 4.20(\mathrm{~d}, J=$ $9.9 \mathrm{~Hz}, 0.3 \mathrm{H}), 4.09$ (d, $J=10.3 \mathrm{~Hz}, 0.7 \mathrm{H}), 4.03(\mathrm{~d}, J=10.4 \mathrm{~Hz}, 0.7 \mathrm{H}), 3.80-3.73$ (m, $0.7 \mathrm{H}), 3.65(\mathrm{~d}, J=9.9 \mathrm{~Hz}, 0.3 \mathrm{H}), 3.55-3.49(\mathrm{~m}, 0.3 \mathrm{H}), 3.24(\mathrm{~d}, J=10.3 \mathrm{~Hz}, 0.7 \mathrm{H})$, 3.10-3.06 (m, 0.3 H), 3.02-2.94 (m, $0.7 \mathrm{H}), 2.85-2.79(\mathrm{~m}, 0.3 \mathrm{H}), 2.72-2.64(\mathrm{~m}, 0.7 \mathrm{H})$, 2.55-2.17 (m, $4.3 \mathrm{H}), 1.04(\mathrm{~s}, 0.9 \mathrm{H}), 1.00(\mathrm{~s}, 2.7 \mathrm{H}), 0.96(\mathrm{~s}, 6.3 \mathrm{H}) ;{ }^{13} \mathrm{C}$ NMR $(125$ $\left.\mathrm{MHz}, \mathrm{CDCl}_{3}\right) \delta 210.0,209.1,142.1,141.5,135.8,135.7,135.7,135.6,133.6,133.6$, $133.3,132.8,129.8,129.7,129.7,129.6,128.5,128.5,128.4,128.4,127.7,127.7,127.6$, $125.9,125.9,117.7,82.8,78.8,77.3,75.9,66.1,64.2,55.9,54.0,45.2,44.3,40.7,40.5$, 33.0, 32.9, 31.4, 31.3, 29.7, 26.8, 19.3, 19.2, 15.4, 15.1; HRMS (EI) calcd for $\left[\mathrm{C}_{34} \mathrm{H}_{42} \mathrm{O}_{3} \mathrm{Si}+\mathrm{Na}\right]^{+}$549.2801, found 549.2789. Anal. Calcd for $\mathrm{C}_{34} \mathrm{H}_{42} \mathrm{O}_{3} \mathrm{Si}: \mathrm{C}, 77.52 ; \mathrm{H}$ 8.04. Found: C, 77.70; H, 7.99.

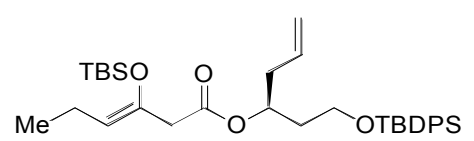

3-(tert-Butyldimethylsilanyloxy)hex-3-enoic acid 1-[2-(tert-butyldiphenylsilanyloxy)ethyl]-but-3-enyl ester (112). A sample of $100 \mathrm{mg}$ of 8 was converted to 94 $\mathrm{mg}(82 \%)$ of 112 , isolated as a single $Z$-isomer (NOE): $[\alpha]^{22}{ }_{\mathrm{D}}+11.3\left(c 0.53, \mathrm{CHCl}_{3}\right)$; IR (neat) 2931, 1738, 1428, 1112, $825 \mathrm{~cm}^{-1} ;{ }^{1} \mathrm{H}$ NMR (400 MHz, $\left.\mathrm{C}_{6} \mathrm{D}_{6}\right) \delta 7.81-7.74(\mathrm{~m}, 4$ H), 7.29-7.20 (m, $6 \mathrm{H}), 5.75$ (dddd, $J=16.0,11.4,7.0,7.0 \mathrm{~Hz}, 1 \mathrm{H}), 5.36-5.29(\mathrm{~m}, 1 \mathrm{H})$, 5.02-4.96 (m, $2 \mathrm{H}), 4.57$ (t, $J=7.1 \mathrm{~Hz}, 1 \mathrm{H}), 3.79-3.68$ (m, $2 \mathrm{H}), 2.94$ (s, $2 \mathrm{H}), 2.35-2.20$ (m, 2 H), 2.16-2.07 (m, 2 H), 1.86-1.73 (m, 2 H), 1.18 (s, 9 H), 0.99 (s, $1 \mathrm{H}), 0.94$ (t, $J=$ 7.6 Hz, $3 \mathrm{H}), 0.14$ (s, $3 \mathrm{H}), 0.13$ (s, $3 \mathrm{H}) ;{ }^{13} \mathrm{C}$ NMR $\left(100 \mathrm{MHz}, \mathrm{C}_{6} \mathrm{D}_{6}\right) \delta 169.8,143.9$, 
136.4, 136.4, 134.5, 134.4, 130.4, 128.6, 128.5, 118.1, 114.4, 71.3, 60.9, 43.5, 39.2, 37.0, 27.4, 26.3, 19.8, 19.6, 18.8, 14.8, -3.6, -3.6; HRMS (EI) calcd for $\left[\mathrm{C}_{34} \mathrm{H}_{52} \mathrm{O}_{4} \mathrm{Si}_{2}+\mathrm{Na}\right]^{+}$ 603.3302, found 603.3301.

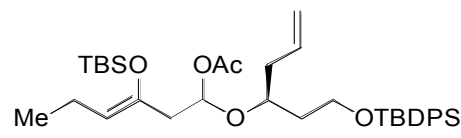

Acetic acid 3-(tert-butyldimethylsilanyloxy)-1-\{1-[2-(tert-butyldiphenylsilanyloxy)ethyl]but-3-enyloxy-hex-3-enyl ester (113). A sample of $52 \mathrm{mg}$ of $\mathbf{1 1 2}$ was converted to $55 \mathrm{mg}(98 \%)$ of 113 , isolated as a 1:1 mixture of diastereomers: $[\mathrm{a}]^{22}{ }_{\mathrm{D}}+8.3(c 0.855$, $\mathrm{CHCl}_{3}$ ); IR (neat) 2931, 1742, 1428, 1007, $825 \mathrm{~cm}^{-1} ;{ }^{1} \mathrm{H} \mathrm{NMR}\left(400 \mathrm{MHz}, \mathrm{C}_{6} \mathrm{D}_{6}\right) \delta 7.82-$ 7.75 (m, 4 H), 7.24-7.20 (m, 6 H), 6.48-6.43 (m, 1 H), 6.00-5.84 (m, 1 H), 5.09-4.98 (m, $2 \mathrm{H}), 4.57(\mathrm{t}, J=7.1 \mathrm{~Hz}, 0.5 \mathrm{H}), 4.56(\mathrm{t}, J=7.1 \mathrm{~Hz}, 0.5 \mathrm{H}), 4.12-4.03(\mathrm{~m}, 1 \mathrm{H})$, 3.96-3.78 (m, 2 H), 2.55-2.50 (m, 2 H), 2.41-2.27 (m, 2 H), 2.16-2.05 (m, 2 H), 1.981.75 (m, 2 H), 1.73 (s, $1.5 \mathrm{H}), 1.73$ (s, $1.5 \mathrm{H}), 1.72$ (s, $1.5 \mathrm{H}), 1.20$ (s, $4.5 \mathrm{H}), 1.17$ (s, 4.5 H), 1.01 (s, $9 \mathrm{H}), 0.94$ (t, $J=7.5 \mathrm{~Hz}, 1.5 \mathrm{H}), 0.94$ (t, $J=7.5 \mathrm{~Hz}, 1.5 \mathrm{H}), 0.15(\mathrm{~s}, 3 \mathrm{H})$, $0.12(\mathrm{~s}, 1.5 \mathrm{H}), 0.12(\mathrm{~s}, 1.5 \mathrm{H}) ;{ }^{13} \mathrm{C} \mathrm{NMR}\left(100 \mathrm{MHz}, \mathrm{C}_{6} \mathrm{D}_{6}\right) \delta 170.5,170.3,145.3,145.2$, $136.4,136.4,136.4,136.3,135.6,134.9,134.8,134.6,130.3,130.3,128.6,117.9,117.3$, 114.3, 114.1, 96.3, 95.2, 77.0, 75.2, 61.4, 61.1, 43.4, 40.1, 39.1, 38.1, 37.7, 27.5, 27.5, 27.1, 26.4, 26.3, 21.3, 19.8, 19.5, 18.8, 18.8, 14.9, -3.4, -3.4, -3.4, -3.6; HRMS (EI) calcd for $\left[\mathrm{C}_{36} \mathrm{H}_{56} \mathrm{O}_{5} \mathrm{Si}_{2}+\mathrm{Na}\right]^{+}$647.3564, found 647.3568 .

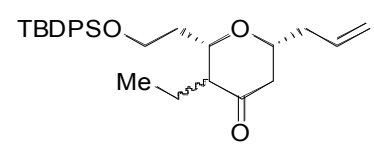

6-Allyl-2-[2-(tert-butyldiphenylsilanyloxy)ethyl]-3-ethyltetrahydropyran-4-one (25). A sample of $36 \mathrm{mg}$ of $\mathbf{1 1 3}$ was converted to $22 \mathrm{mg}(85 \%)$ of $\mathbf{2 5}$, isolated as a 2:1 mixture of C-3 epimers: IR (neat) $2961,1715,1428,1112 \mathrm{~cm}^{-1} ;{ }^{1} \mathrm{H}$ NMR $\left(500 \mathrm{MHz}, \mathrm{C}_{6} \mathrm{D}_{6}\right) \delta$ 
7.79-7.74 (m, 4 H), 7.25-7.23 (m, 6 H), 5.72-5.61 (m, 1 H), 5.00-4.91 (m, 2 H), 4.00 (ddd, $J=9.7,9.7,5.3 \mathrm{~Hz}, 0.5 \mathrm{H}), 3.88-3.70(\mathrm{~m}, 1.5 \mathrm{H}), 3.67$ (ddd, $J=8.9,3.2,3.2 \mathrm{~Hz}$, $0.5 \mathrm{H}), 3.50$ (ddd, $J=10.0,10.0,2.0 \mathrm{~Hz}, 0.5 \mathrm{H}), 3.28-3.20(\mathrm{~m}, 1 \mathrm{H}), 2.20-1.88(\mathrm{~m}, 5 \mathrm{H})$, 1.86-1.78 (m, $1 \mathrm{H}), 1.62-1.42(\mathrm{~m}, 2.5 \mathrm{H}), 1.36-1.28$ (m, $1.5 \mathrm{H}), 1.17$ (s, $4.5 \mathrm{H}), 1.16$ (s, $4.5 \mathrm{H}), 0.96(\mathrm{t}, J=7.4 \mathrm{~Hz}, 1.5 \mathrm{H}), 0.73(\mathrm{t}, J=7.5 \mathrm{~Hz}, 1.5 \mathrm{H}) ;{ }^{13} \mathrm{C} \mathrm{NMR}\left(125 \mathrm{MHz}, \mathrm{C}_{6} \mathrm{D}_{6}\right)$ ઈ 208.7, 206.6, 136.3, 134.4, 134.4, 130.4, 130.4, 128.9, 128.7, 128.6, 128.2, 117.9, $117.8,77.2,77.2,76.6,76.5,61.3,60.9,57.3,56.4,48.1,44.7,41.1,37.7,35.8,27.5$, 19.8, 18.7, 18.0, 12.0; HRMS (EI) calcd for $\left[\mathrm{C}_{28} \mathrm{H}_{38} \mathrm{O}_{3} \mathrm{Si}+\mathrm{Na}\right]^{+}$473.2488, found 472.2491

Epimerization of the C-3 center: Treatment of $\mathbf{2 5}$ with 1:1 aq. $\mathrm{NaOH}(1.0 \mathrm{M}) / \mathrm{THF}$ at reflux enriched the C-3 equatorial product from 1:1 to 3.5:1 (99\% mass recovery).

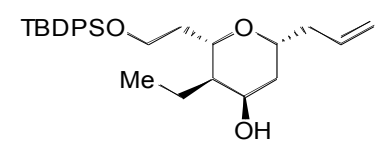

\section{6-Allyl-2-[2-(tert-butyldiphenylsilanyloxy)ethyl]-3-ethyltetrahydropyran-4-ol (26).}

L-Selectride ( $27 \mu \mathrm{L}, 27 \mu$ mol, 1.2 equiv.) was added to a $-78{ }^{\circ} \mathrm{C}$ solution of pure $\mathrm{C}-3$ equatorial 25 ( $8 \mathrm{mg}, 12.8 \mu \mathrm{mol}, 1.0$ equiv) in $0.2 \mathrm{~mL}$ of THF. After $30 \mathrm{~min}, 1$ drop of $\mathrm{MeOH}, 1 \mathrm{~N} \mathrm{NaOH}$ and $30 \% \mathrm{H}_{2} \mathrm{O}_{2}$ were sequentially added and the reaction mixture was allowed to stir with warming to room temperature over $1.5 \mathrm{~h}$. After dilution with $\mathrm{H}_{2} \mathrm{O}$ (2 $\mathrm{mL}$ ), the aqueous was extracted with ethyl acetate $(3 \times 5 \mathrm{~mL})$, then washed with $\mathrm{H}_{2} \mathrm{O}$ and brine. The extracts were dried over anhydrous $\mathrm{MgSO}_{4}$ and concentrated. Purification (20\% EtOAc/hexanes, $\mathrm{R}_{\mathrm{f}}=0.49$ in $30 \%$ EtOAc/hexanes) provided $7 \mathrm{mg}(88 \%)$ of $\mathbf{2 6}$ as a single diastereomer: $[\alpha]_{D}^{22}-7.0\left(c 0.10, \mathrm{CHCl}_{3}\right)$; IR (neat) $3412,2930,1428,1112,701$ $\mathrm{cm}^{-1} ;{ }^{1} \mathrm{H}$ NMR (500 MHz, $\mathrm{CDCl}_{3}$ ) 7.74-7.65 (m, 4 H), 7.44-7.35 (m, $\left.6 \mathrm{H}\right), 5.76$ (dddd, 
$J=17.1,10.0,6.9,6.9 \mathrm{~Hz}, 1 \mathrm{H}), 5.05-4.95(\mathrm{~m}, 2 \mathrm{H}), 4.15-4.12(\mathrm{~m}, 1 \mathrm{H}), 3.88-3.73(\mathrm{~m}, 2$ H), 3.71-3.61 (m, $2 \mathrm{H}), 2.25-2.19(\mathrm{~m}, 1 \mathrm{H}), 2.12-2.05(\mathrm{~m}, 1 \mathrm{H}), 1.97-1.90(\mathrm{~m}, 1 \mathrm{H})$, 1.79-1.75 (m, 1 H), 1.48-1.39 (m, 2 H), 1.29-1.16 (m, 4 H), 1.07 (s, 9 H), 0.93 (t, $J=7.3$ $\mathrm{Hz}, 3 \mathrm{H}) ;{ }^{13} \mathrm{C} \mathrm{NMR}\left(125 \mathrm{MHz}, \mathrm{CDCl}_{3}\right) \delta 141.7,135.6,134.8,129.7,129.4,127.7,127.5$, $120.9,116.4,72.5,70.4,66.5,65.0,60.5,51.8,45.8,40.4,39.2,36.0,26.9,26.5,19.3$, 11.0; HRMS (EI) calcd for $\left[\mathrm{C}_{28} \mathrm{H}_{40} \mathrm{O}_{3} \mathrm{Si}+\mathrm{Na}\right]^{+}$475.2644, found 475.2653.

Advanced Mosher analysis of the $R$ - and $S$-MTPA esters:

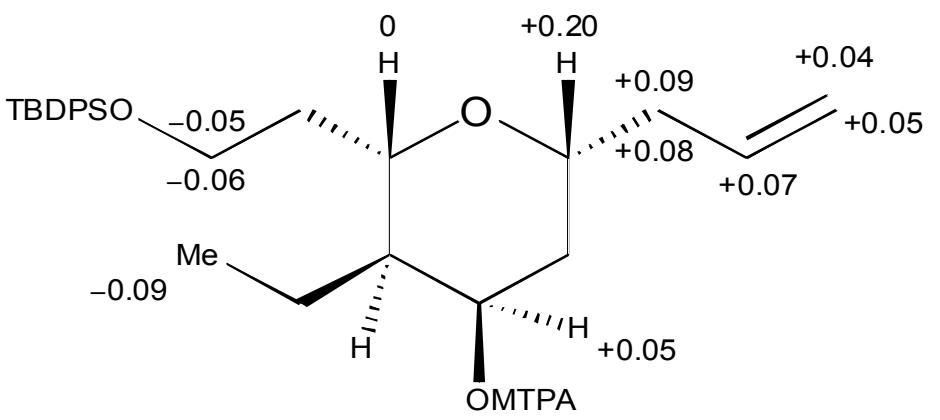

$\delta S-\delta R$ values (ppm) 